Network Working Group

Request for Comments: 5065

Obsoletes: 3065

Category: Standards Track
P. Traina

Blissfully Retired

D. McPherson

Arbor Networks

J. Scudder

Juniper Networks

August 2007

\title{
Autonomous System Confederations for BGP
}

Status of This Memo

This document specifies an Internet standards track protocol for the Internet community, and requests discussion and suggestions for improvements. Please refer to the current edition of the "Internet Official Protocol Standards" (STD 1) for the standardization state and status of this protocol. Distribution of this memo is unlimited.

Copyright Notice

Copyright (C) The IETF Trust (2007).

Abstract

The Border Gateway Protocol (BGP) is an inter-autonomous system routing protocol designed for Transmission Control Protocol/Internet Protocol (TCP/IP) networks. BGP requires that all BGP speakers within a single autonomous system (AS) must be fully meshed. This represents a serious scaling problem that has been well documented in a number of proposals.

This document describes an extension to BGP that may be used to create a confederation of autonomous systems that is represented as a single autonomous system to BGP peers external to the confederation, thereby removing the "full mesh" requirement. The intention of this extension is to aid in policy administration and reduce the management complexity of maintaining a large autonomous system.

This document obsoletes RFC 3065 . 
Table of Contents

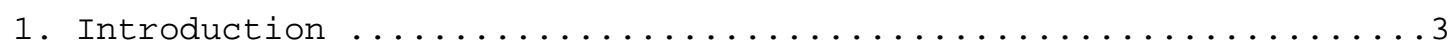

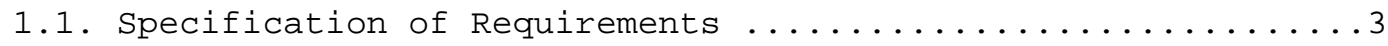

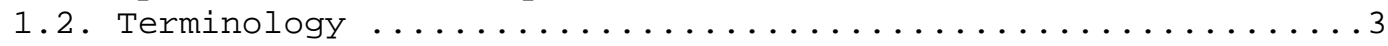

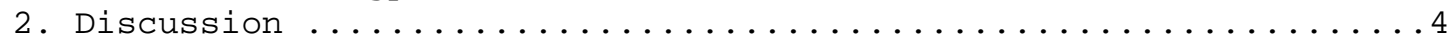

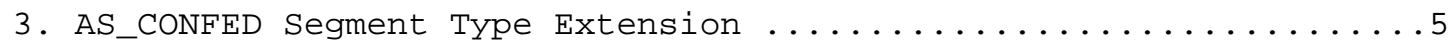

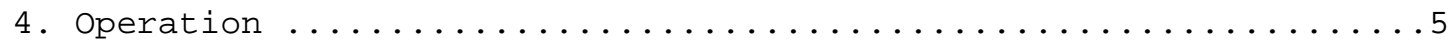

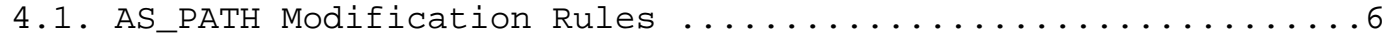

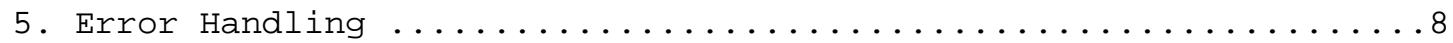

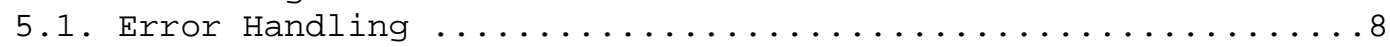

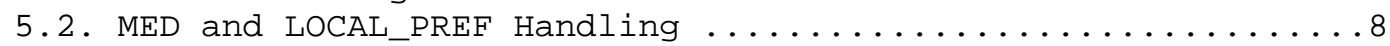

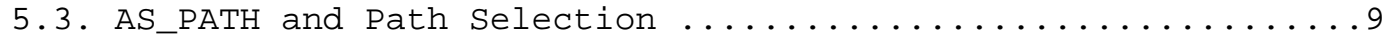

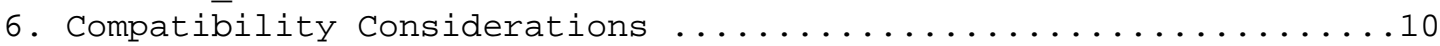

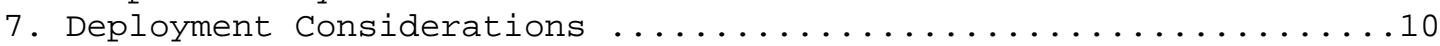

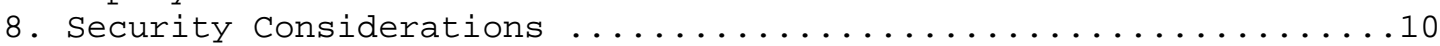

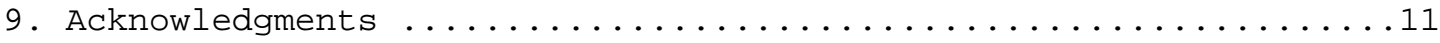

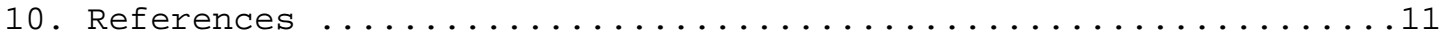

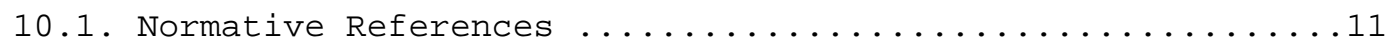

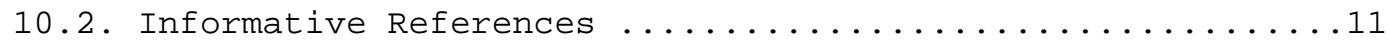

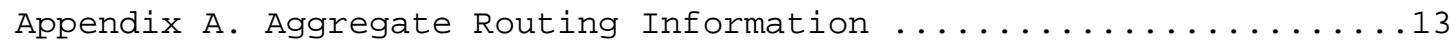

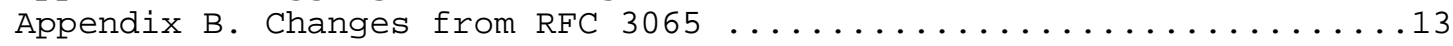




\section{Introduction}

As originally defined, BGP requires that all BGP speakers within a single AS must be fully meshed. The result is that for $n$ BGP speakers within an AS, $n *(n-1) / 2$ unique Internal BGP (IBGP) sessions are required. This "full mesh" requirement clearly does not scale when there are a large number of IBGP speakers within the autonomous system, as is common in many networks today.

This scaling problem has been well documented and a number of proposals have been made to alleviate this, such as [RFC2796] and [RFC1863] (made historic by [RFC4223]). This document presents another alternative alleviating the need for a "full mesh" and is known as "Autonomous System Confederations for BGP", or simply, "BGP confederations". It has also been observed that BGP confederations may provide improvements in routing policy control.

This document is a revision of, and obsoletes, [RFC3065], which is itself a revision of [RFC1965]. It includes editorial changes, terminology clarifications, and more explicit protocol specifications based on extensive implementation and deployment experience with BGP Confederations.

\subsection{Specification of Requirements}

The key words "MUST", "MUST NOT", "REQUIRED", "SHALL", "SHALL NOT", "SHOULD", "SHOULD NOT", "RECOMMENDED", "MAY", and "OPTIONAL" in this document are to be interpreted as described in RFC 2119 [RFC2119].

\subsection{Terminology}

AS Confederation

A collection of autonomous systems represented and advertised as a single AS number to BGP speakers that are not members of the local BGP confederation.

\section{AS Confederation Identifier}

An externally visible autonomous system number that identifies a BGP confederation as a whole.

Member Autonomous System (Member-AS)

An autonomous system that is contained in a given AS confederation. Note that "Member Autonomous System" and "MemberAS" are used entirely interchangeably throughout this document. 
Member-AS Number

An autonomous system number identifier visible only within a BGP confederation, and used to represent a Member-AS within that confederation.

2. Discussion

It may be useful to subdivide autonomous systems with a very large number of BGP speakers into smaller domains for purposes of controlling routing policy via information contained in the BGP AS_PATH attribute. For example, one may choose to consider all BGP speakers in a geographic region as a single entity.

In addition to potential improvements in routing policy control, if techniques such as those presented here or in [RFC4456] are not employed, [BGP-4] requires BGP speakers in the same autonomous system to establish a full mesh of TCP connections among all speakers for the purpose of exchanging exterior routing information. In autonomous systems, the number of intra-domain connections that need to be maintained by each border router can become significant.

Subdividing a large autonomous system allows a significant reduction in the total number of intra-domain BGP connections, as the connectivity requirements simplify to the model used for inter-domain connections.

Unfortunately, subdividing an autonomous system may increase the complexity of routing policy based on AS_PATH information for all members of the Internet. Additionally, this division increases the maintenance overhead of coordinating external peering when the internal topology of this collection of autonomous systems is modified.

Therefore, division of an autonomous system into separate systems may adversely affect optimal routing of packets through the Internet.

However, there is usually no need to expose the internal topology of this divided autonomous system, which means it is possible to regard a collection of autonomous systems under a common administration as a single entity or autonomous system, when viewed from outside the confines of the confederation of autonomous systems itself. 


\section{AS_CONFED Segment Type Extension}

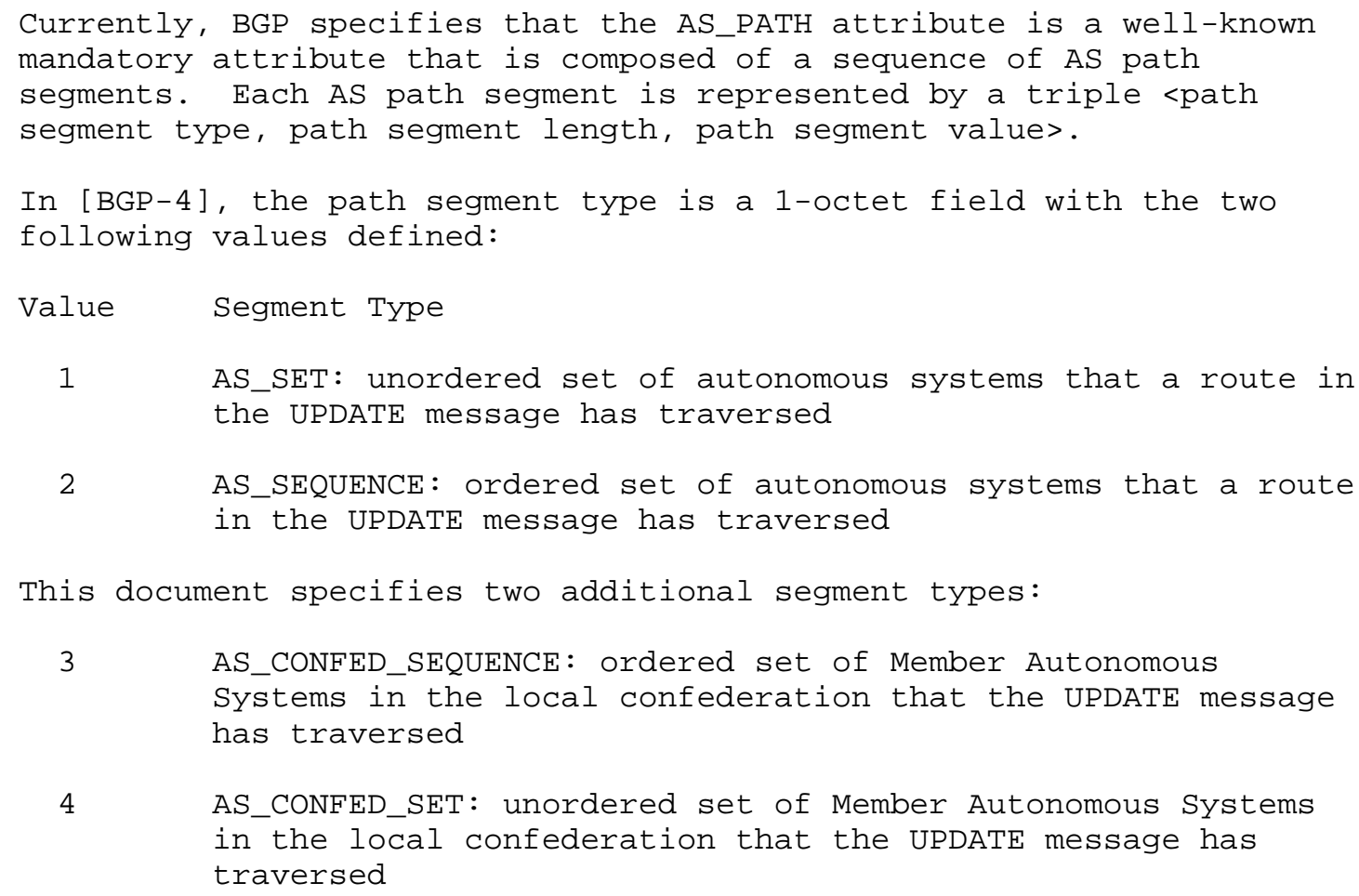

4. Operation

A member of a BGP confederation MUST use its AS Confederation Identifier in all transactions with peers that are not members of its confederation. This AS Confederation Identifier is the "externally visible" AS number, and this number is used in OPEN messages and advertised in the AS_PATH attribute.

A member of a BGP confederation MUST use its Member-AS Number in all transactions with peers that are members of the same confederation as the local BGP speaker.

A BGP speaker receiving an AS_PATH attribute containing an autonomous system matching its own AS Confederation Identifier SHALL treat the path in the same fashion as if it had received a path containing its own AS number. 
A BGP speaker receiving an AS_PATH attribute containing an AS_CONFED_SEQUENCE or AS_CONEED_SET that contains its own Member-AS Number SHALL treat the path in the same fashion as if it had received a path containing its own AS number.

\subsection{AS_PATH Modification Rules}

When implementing BGP confederations, section 5.1 .2 of [BGP-4] is replaced with the following text:

AS_PATH is a well-known mandatory attribute. This attribute identifies the autonomous systems through which routing information carried in this UPDATE message has passed. The components of this list can be AS_SETS, AS_SEQUENCES, AS_CONFED_SETS or AS_CONFED_SEQUENCES.

When a BGP speaker propagates a route it learned from another BGP speaker's UPDATE message, it modifies the route's AS_PATH attribute based on the location of the BGP speaker to which the route will be sent:

a) When a given BGP speaker advertises the route to another BGP speaker located in its own Member-AS, the advertising speaker SHALL NOT modify the AS_PATH attribute associated with the route.

b) When a given BGP speaker advertises the route to a BGP speaker located in a neighboring autonomous system that is a member of the local confederation, the advertising speaker updates the AS_PATH attribute as follows:

1) if the first path segment of the AS_PATH is of type AS_CONFED_SEQUENCE, the local system prepends its own Member-AS number as the last element of the sequence (put it in the leftmost position with respect to the position of octets in the protocol message). If the act of prepending will cause an overflow in the AS_PATH segment (i.e., more than 255 ASs), it SHOULD prepend a new segment of type AS_CONFED_SEQUENCE and prepend its own AS number to this new segment.

2) if the first path segment of the AS_PATH is not of type AS_CONFED_SEQUENCE, the local system prepends a new path segment of type AS_CONFED_SEQUENCE to the AS_PATH, including its own Member-AS Number in that segment.

3) if the AS_PATH is empty, the local system creates a path segment of type AS_CONFED_SEQUENCE, places its own Member-AS Number into that segment, and places that segment into the AS_PATH. 
c) When a given BGP speaker advertises the route to a BGP speaker located in a neighboring autonomous system that is not a member of the local confederation, the advertising speaker SHALL update the AS_PATH attribute as follows:

1) if any path segments of the AS_PATH are of the type AS_CONFED_SEQUENCE or AS_CONFED_SET, those segments MUST be removed from the AS_PATH attribute, leaving the sanitized AS_PATH attribute to be operated on by steps 2, 3 or 4 .

2) if the first path segment of the remaining AS_PATH is of type AS_SEQUENCE, the local system prepends its own AS Confederation Identifier as the last element of the sequence (put it in the leftmost position with respect to the position of octets in the protocol message). If the act of prepending will cause an overflow in the AS_PATH segment (i.e., more than 255 ASs), it SHOULD prepend a new segment of type AS_SEQUENCE and prepend its own AS number to this new segment.

3) if the first path segment of the remaining AS_PATH is of type AS_SET, the local system prepends a new path segment of type AS_SEQUENCE to the AS_PATH, including its own AS Confederation Identifier in that segment.

4) if the remaining AS_PATH is empty, the local system creates a path segment of type AS_SEQUENCE, places its own AS Confederation Identifier into that segment, and places that segment into the AS_PATH.

When a BGP speaker originates a route then:

a) the originating speaker includes its own AS Confederation Identifier in a path segment, of type AS_SEQUENCE, in the AS_PATH attribute of all UPDATE messages sent to BGP speakers located in neighboring autonomous systems that are not members of the local confederation. In this case, the AS Confederation Identifier of the originating speaker's autonomous system will be the only entry the path segment, and this path segment will be the only segment in the AS_PATH attribute.

b) the originating speaker includes its own Member-AS Number in a path segment, of type AS_CONEED_SEQUENCE, in the AS_PATH attribute of all UPDATE messages sent to BGP speakers located in neighboring Member Autonomous systems that are members of the local confederation. In this case, the Member-AS Number of the originating speaker's autonomous system will be the only entry the path segment, and this path segment will be the only segment in the AS_PATH attribute. 
c) the originating speaker includes an empty AS_PATH attribute in all UPDATE messages sent to BGP speakers residing within the same Member-AS. (An empty AS_PATH attribute is one whose length field contains the value zero).

Whenever the modification of the AS_PATH attribute calls for including or prepending the AS Confederation Identifier or Member-AS Number of the local system, the local system MAY include/prepend more than one instance of that value in the AS_PATH attribute. This is controlled via local configuration.

5. Error Handling

A BGP speaker MUST NOT transmit updates containing AS_CONFED_SET or AS_CONFED_SEQUENCE attributes to peers that are not members of the local confederation.

It is an error for a BGP speaker to receive an UPDATE message with an AS_PATH attribute that contains AS_CONFED_SEQUENCE or AS_CONFED_SET segments from a neighbor that is not located in the same confederation. If a BGP speaker receives such an UPDATE message, it SHALL treat the message as having a malformed AS_PATH according to the procedures of [BGP-4], Section 6.3 ("UPDATE Message Error Handling") .

It is a error for a BGP speaker to receive an update message from a confederation peer that is not in the same Member-AS that does not have AS_CONFED_SEQUENCE as the first segment. If a BGP speaker receives such an UPDATE message, it SHALL treat the message as having a malformed AS_PATH according to the procedures of [BGP-4], Section 6.3 ("UPDATE Message Error Handling").

5.1. Common Administrative Issues

It is reasonable for Member Autonomous systems of a confederation to share a common administration and Interior Gateway Protocol (IGP) information for the entire confederation. It is also reasonable for each Member-AS to run an independent IGP. In the latter case, the NEXT_HOP may need to be set using policy (i.e., by default it is unchanged).

5.2. MED and LOCAL_PREF Handling

It SHALL be legal for a BGP speaker to advertise an unchanged NEXT_HOP and MULTI_EXIT_DISC (MED) attribute to peers in a neighboring Member-AS of the local confederation. 
MEDs of two routes SHOULD only be compared if the first autonomous systems in the first AS_SEQUENCE in both routes are the same -- i.e., skip all the autonomous systems in the AS_CONFED_SET and AS_CONFED_SEQUENCE. An implementation MAY provide the ability to configure path selection such that MEDs of two routes are comparable if the first autonomous systems in the AS_PATHs are the same, regardless of AS_SEQUENCE or AS_CONFED_SEQUENCE in the AS_PATH.

An implementation MAY compare MEDs received from a Member-AS via multiple paths. An implementation MAY compare MEDs from different Member Autonomous systems of the same confederation.

In addition, the restriction against sending the LOCAL_PREF attribute to peers in a neighboring autonomous system within the same confederation is removed.

\subsection{AS_PATH and Path Selection}

Path selection criteria for information received from members inside a confederation MUST follow the same rules used for information received from members inside the same autonomous system, as specified in $[B G P-4]$.

In addition, the following rules SHALL be applied:

1) If the AS_PATH is internal to the local confederation (i.e., there are only AS_CONFED_* segments), consider the neighbor AS to be the local AS.

2) Otherwise, if the first segment in the path that is not an AS_CONFED_SEQUENCE or AS_CONFED_SET is an AS_SEQUENCE, consider the neighbor AS to be the leftmost AS_SEQUENCE AS.

3) When comparing routes using AS_PATH length, CONFED_SEQUENCE and CONFED_SETS SHOULD NOT be counted.

4) When comparing routes using the internal (IBGP learned) versus external (EBGP learned) rules, treat a route that is learned from a peer that is in the same confederation (not necessarily the same Member-AS) as "internal". 


\section{Compatibility Considerations}

All BGP speakers participating as members of a confederation MUST recognize the AS_CONFED_SET and AS_CONFED_SEQUENCE segment type extensions to the AS_PATH attribute.

Any BGP speaker not supporting these extensions will generate a NOTIFICATION message specifying an "UPDATE Message Error" and a subcode of "Malformed AS_PATH".

This compatibility issue implies that all BGP speakers participating in a confederation MUST support BGP confederations. However, BGP speakers outside the confederation need not support these extensions.

7. Deployment Considerations

BGP confederations have been widely deployed throughout the Internet for a number of years and are supported by multiple vendors.

Improper configuration of BGP confederations can cause routing information within an AS to be duplicated unnecessarily. This duplication of information will waste system resources, cause unnecessary route flaps, and delay convergence.

Care should be taken to manually filter duplicate advertisements caused by reachability information being relayed through multiple Member Autonomous systems based upon the topology and redundancy requirements of the confederation.

Additionally, confederations (as well as route reflectors), by excluding different reachability information from consideration at different locations in a confederation, have been shown [RFC3345] to cause permanent oscillation between candidate routes when using the tie-breaking rules required by BGP [BGP-4]. Care must be taken when selecting MED values and tie-breaking policy to avoid these situations.

One potential way to avoid this is by configuring inter-Member-AS IGP metrics higher than intra-Member-AS IGP metrics and/or using other tie-breaking policies to avoid BGP route selection based on incomparable MEDs.

8. Security Considerations

This extension to BGP does not change the underlying security issues inherent in the existing BGP protocol, such as those described in [RFC2385] and [BGP-VULN]. 


\section{Acknowledgments}

The general concept of BGP confederations was taken from IDRP's Routing Domain Confederations [ISO10747]. Some of the introductory text in this document was taken from [RFC2796].

The authors would like to acknowledge Jeffrey Haas for his extensive feedback on this document. We'd also like to thank Bruce Cole, Srihari Ramachandra, Alex Zinin, Naresh Kumar Paliwal, Jeffrey Haas, Cengiz Alaettinoglu, Mike Hollyman, and Bruno Rijsman for their feedback and suggestions.

Finally, we'd like to acknowledge Ravi Chandra and Yakov Rekhter for providing constructive and valuable feedback on earlier versions of this specification.

10. References

10.1. Normative References

[BGP-4] Rekhter, Y., Ed., Li, T., Ed., and S. Hares, Ed., "A Border Gateway Protocol 4 (BGP-4)", RFC 4271, January 2006 .

[RFC1965] Traina, P., "Autonomous System Confederations for BGP", RFC 1965, June 1996.

[RFC2119] Bradner, S., "Key words for use in RFCs to Indicate Requirement Levels", BCP 14, RFC 2119, March 1997.

[RFC3065] Traina, P., MCPherson, D., and J. Scudder, "Autonomous System Confederations for BGP", RFC 3065, February 2001.

\subsection{Informative References}

[ISO10747] Kunzinger, C., Editor, "Inter-Domain Routing Protocol", ISO/IEC 10747, October 1993.

[RFC1863] Haskin, D., "A BGP/IDRP Route Server alternative to a full mesh routing", RFC 1863, October 1995.

[RFC2385] Heffernan, A., "Protection of BGP Sessions via the TCP MD5 Signature Option", RFC 2385, August 1998.

[RFC3345] McPherson, D., Gill, V., Walton, D., and A. Retana, "Border Gateway Protocol (BGP) Persistent Route Oscillation Condition", RFC 3345, August 2002. 
[RFC4223] Savola, P., "Reclassification of RFC 1863 to Historic", RFC 4223, October 2005 .

[RFC4272] Murphy, S., "BGP Security Vulnerabilities Analysis", RFC 4272, January 2006 .

[RFC4456] Bates, T., Chen, E., and R. Chandra, "BGP Route Reflection: An Alternative to Full Mesh Internal BGP (IBGP)", RFC 4456, April 2006. 
Appendix A. Aggregate Routing Information

As a practical matter, aggregation as discussed in [BGP-4], Section 9.2.2.2, is not generally employed within confederations. However, in the event that such aggregation is performed within a confederation, the rules of [BGP-4] should be followed, making the necessary substitutions between AS_SET and AS_CONFED_SET and similarly, AS_SEQUENCE and AS_CONFED_SEQUENCE. Confederation-type segments (AS_CONFED_SET and AS_CONFED_SEQUENCE) MUST be kept separate from non-confederation segments (AS_SET and AS_SEQUENCE). An implementation could also choose to provide a form of aggregation wherein non-confederation segments are aggregated as discussed in [BGP-4], Section 9.2.2.2, and confederation-type segments are not aggregated.

Support for aggregation of confederation-type segments is not mandatory .

Appendix B. Changes from RFC 3065

The primary trigger for an update to RFC 3065 was regarding issues associated with AS path segment handling, in particular what to do when interacting with BGP peers external to a confederation and to ensure AS_CONFED_[SET|SEQUENCE] segment types are not propagated to peers outside of a confederation.

As such, the "Error Handling" section above was added and applies not only to BGP confederation speakers, but to all BGP speakers.

Other changes are mostly trivial and surrounding some clarification and consistency in terminology and denoting that AS_CONFED_[SET|SEQUENCE] Segment Type handling should be just as it is in the base BGP specification [BGP-4].

Authors' Addresses

Paul Traina

Blissfully Retired

Email: bgp-confederationsest04.pst.org

Danny McPherson

Arbor Networks

EMail: danny@arbor. net

John G. Scudder

Juniper Networks

EMail: jgs@juniper.net 
Full Copyright statement

Copyright (C) The IETF Trust (2007).

This document is subject to the rights, licenses and restrictions contained in BCP 78, and except as set forth therein, the authors retain all their rights.

This document and the information contained herein are provided on an "AS IS" basis and THE CONTRIBUTOR, THE ORGANIZATION HE/SHE REPRESENTS OR IS SPONSORED BY (IF ANY), THE INTERNET SOCIETY, THE IETF TRUST AND THE INTERNET ENGINEERING TASK FORCE DISCLAIM ALL WARRANTIES, EXPRESS OR IMPLIED, INCLUDING BUT NOT LIMITED TO ANY WARRANTY THAT THE USE OF THE INFORMATION HEREIN WILL NOT INFRINGE ANY RIGHTS OR ANY IMPLIED WARRANTIES OF MERCHANTABILITY OR FITNESS FOR A PARTICULAR PURPOSE.

Intellectual Property

The IETF takes no position regarding the validity or scope of any Intellectual Property Rights or other rights that might be claimed to pertain to the implementation or use of the technology described in this document or the extent to which any license under such rights might or might not be available; nor does it represent that it has made any independent effort to identify any such rights. Information on the procedures with respect to rights in RFC documents can be found in BCP 78 and BCP 79 .

Copies of IPR disclosures made to the IETF Secretariat and any assurances of licenses to be made available, or the result of an attempt made to obtain a general license or permission for the use of such proprietary rights by implementers or users of this specification can be obtained from the IETF on-line IPR repository at http://www.ietf.org/ipr.

The IETF invites any interested party to bring to its attention any copyrights, patents or patent applications, or other proprietary rights that may cover technology that may be required to implement this standard. Please address the information to the IETF at ietf-ipreietf.org.

Acknowledgement

Funding for the RFC Editor function is currently provided by the Internet Society. 